\title{
The pathophysiology of common causes of syncope
}

\author{
W Arthur, G C Kaye
}

This is the first of three papers on syncope; the second and third papers will be published in fanuary and February respectively.
Cardiology

Department, Castle Hill Hospital, Castle

Road, Cottingham,

East Yorkshire

HU16 5JQ, UK

W Arthur

G C Kaye

Correspondence to: Dr Arthur

Submitted 30 December 1999

Accepted 16 February 2000
Syncope is a transient loss of consciousness secondary to inadequate cerebral perfusion with oxygenated blood. It is a common medical problem, accounting for around $5 \%$ of acute medical admissions and 3\% of emergency department visits. ${ }^{1}$ Syncope secondary to cardiac causes carries the worst prognosis, with a one year mortality rate of $20-30 \% .^{2}$ An understanding of the events preceding syncope is essential if the correct diagnostic strategy is to be implemented.

\section{General pathophysiological concepts}

A state of consciousness is maintained by adequate cerebral blood flow. Cerebral vascular autoregulation ensures that the cerebral blood flow is kept within a narrow range, independent of the underlying systemic blood pressure. In a young healthy adult the systolic blood pressure may fall to $70 \mathrm{~mm} \mathrm{Hg}$ without significant cerebral ischaemia. ${ }^{3}$ Elderly people and those with chronic hypertension are susceptible to relatively small falls in systemic blood pressure, leading to an increased incidence of syncope in this population. ${ }^{4}$

The term "vasovagal" as applied to syncope has been used since the early 1900s and has become synonymous with the common "faint". ${ }^{5}$ Early studies found that vasovagal syncope was the most common cause of fainting, being found in $58 \%$ of patients who had

\section{Box 1: Neurally mediated reflex syncopal syndromes}

- Vasovagal (emotional, common) faint

- Carotid sinus syncope

- Neurocardiogenic syncope (head up tilt/gravitational syncope)

- Increased intrathoracic pressure Cough syncope

Sneeze syncope

Trumpet player's syncope

Weight lifter's syncope

Mess Trick syncope

Valsalva induced syncope

- Postmicturition syncope

- Gastrointestinal stimulation syncope Rectal examination Defaecation syncope Gastrointestinal instrumentation

- Oesophageal/nasopharyngeal stimulation Swallow syncope Glossopharyngeal neuralgia

- Diving reflex

- Drug induced syncope Glyceryl trinitrate Isoprenaline

[reproduced from reference 7, with permission] experienced this symptom. ${ }^{6}$ As our understanding of human neuroautonomic regulation has evolved it has become apparent that the vasovagal episode, although the most common, is one of a number of neurally mediated syncopal syndromes.

\section{Neurally mediated syncopal syndromes}

Neurally mediated syncope can be classified into several distinct syndromes (box 1). These are all associated with acute vasodilatation of the arterial and venous beds and relative or absolute bradycardia. All of the neurally mediated syncopal syndromes involve an inappropriate reflex with afferent, central, and efferent pathways. ${ }^{7}$ During tilt table testing, the triggers for vasovagal syncope are thought to arise from the heart. ${ }^{8}$ As a result, the term "neurocardiogenic syncope" has been used to define one of the commonest responses found during tilt testing (fig 1). ${ }^{9}$

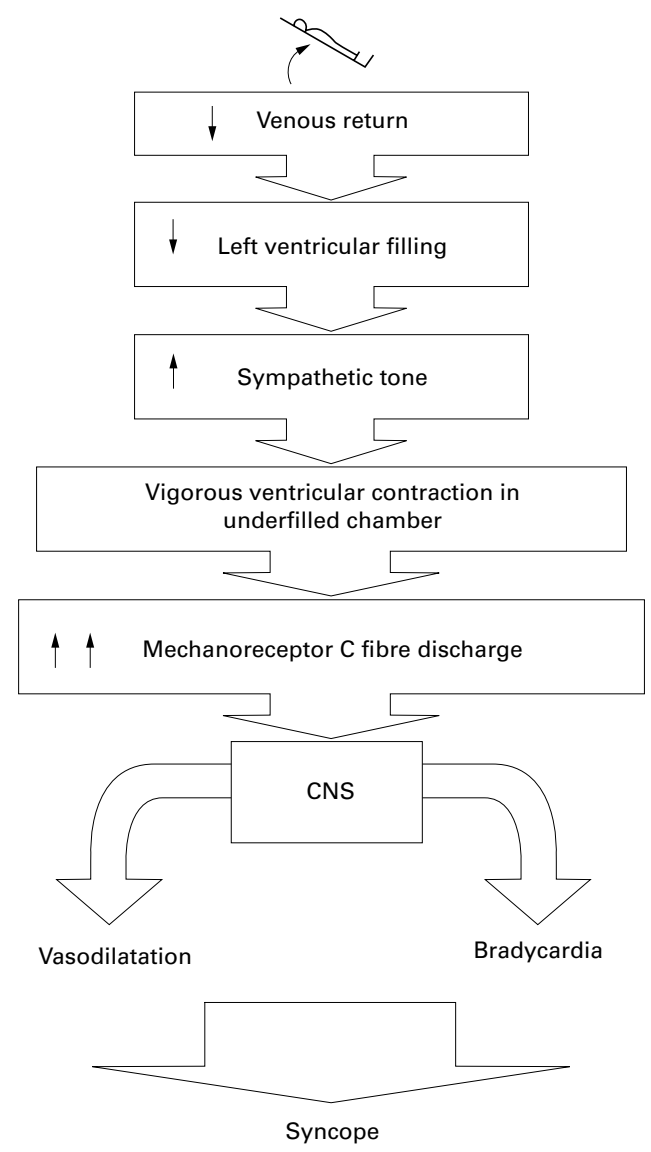

Figure 1 Haemodynamic and autonomic changes characteristic of neurocardiogenic syncope. (Reproduced by permission from Advanstar Communications Inc, as reprinted from Neurology 1995;45(suppl 5):15.

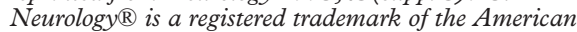
Academy of Neurology.) 
NEUROCARDIOGENIC SYNCOPE

In order to maintain adequate cerebral blood flow in the upright position, man has evolved a series of autonomic reflexes. On standing, 300 to $800 \mathrm{ml}$ of blood shift from the thorax to the lower extremities. This lowers the venous return and hence the cardiac output. Normally this leads to reduced stimulation of baroreceptors in the carotid sinus and aortic arch and mechanoreceptors (vagal C fibres) in the wall of the left ventricle. These receptors inhibit the brain stem neurones responsible for sympathetic stimulation; they also promote the neurones in charge of parasympathetic drive. The end result is increased sympathetic tone and maintenance of the blood pressure. Conversely a rise in blood pressure or intravascular volume increases baroreceptor and mechanoreceptor output, which leads to sympathetic withdrawal and parasympathetic stimulation. ${ }^{10}$

It is thought that the mechanoreceptors in the left ventricle are not only innervated by stretch but also by vigorous and forceful systolic contraction. In patients with neurocardiogenic syncope, overzealous left ventricular contraction occurs in response to reduced venous return. Hence the afferent signals from the left ventricle override the baroreceptor responses, leading to an inappropriate decrease in sympathetic tone and an increase in parasympathetic (vagal) tone. Paradoxically the clinical picture is one of sudden hypotension reflecting diminished sympathetic vasoconstrictor tone accompanied by vagally induced inappropriate bradycardia.

The other forms of neurally mediated syncope share with neurocardiogenic syncope a triggering event stimulating adrenergic tone, followed by a clinical prodrome of vagal overactivation and then sympathetic withdrawal. In the case of the emotionally induced vasovagal faint, higher cortical sites are the predominant triggers of the afferent limb of the reflex arc, which result in increased sympathetic nervous system stimulation. This is then followed by the presyncopal or aura phase characterised by diaphoresis, epigastric discomfort, dizziness/vertigo, and nausea. These prodromal symptoms are induced by the increased parasympathetic tone. They may last anywhere from less than one second to several minutes. These events proceed to syncope unless the subject lies supine or removes the triggering stimulus. The lack of warning signs does not exclude the possibility of neurocardiogenic syncope; a "malignant" form has been described in which there is a rapid deterioration in the haemodynamic state. ${ }^{11}$

The haemodynamic responses evoked in neurally mediated syncope may be predominantly vasodepressor, cardioinhibitory, or mixed. The mixed response is most common, although the vasodepressor component of the mixed response appears to be the dominant factor in up to $85 \%$ of affected patients. ${ }^{12}$ In the malignant type of neurocardiogenic syncope the cardioinhibitory component is pronounced.
CAROTID SINUS HYPERSENSITIVITY AND CAROTID SINUS SYNCOPE

Pressure exerted on the internal carotid artery just above the bifurcation of the common carotid artery leads to slowing of the sinus rate and impaired atrioventricular node conduction. This is a normal response resulting from stimulation of the carotid sinus. In carotid sinus hypersensitivity this reflex is exaggerated and is described in three forms: cardioinhibitory, giving rise to asystole of three seconds or more; vasodepressor, leading to a fall in systolic blood pressure of $50 \mathrm{~mm} \mathrm{Hg}$ or more; or the response may be mixed. ${ }^{13}$ Studies have shown that $5-25 \%$ of asymptomatic older men have carotid sinus hypersensitivity. ${ }^{14}$ At the same time only $5-20 \%$ of patients showing carotid sinus hypersensitivity actually have syncope of carotid sinus origin. ${ }^{14}$ Diagnosis of carotid sinus syncope requires that spontaneous symptoms of presyncope or syncope be reproduced by carotid sinus massage.

Despite the fact that the cardioinhibitory response is dominant, a vasodepressor component of the syndrome can be elicited by carotid sinus massage in the majority of patients with carotid sinus syncope. ${ }^{15}$ The vasodepressor reflex in these patients peaks at the end of carotid sinus massage and may last for up to two minutes. This is in contrast to the cardioinhibitory response, in which the maximum asystolic pause normally occurs within a few beats of the application of carotid sinus massage.

Orthostatic syncope and dysautonomic disturbances of blood pressure control Orthostatic syncope results from the venous pooling of blood that occurs upon changing from a supine to an upright position. There is no vagal hyperactivity associated with this venous pooling and this distinguishes orthostatic syncope from neurocardiogenic syncope. Orthostatic syncope may be the consequence of transient or chronic volume depletion or abnormal vasomotor compensatory mechanisms. Owing to a relative lack of intravascular volume, the patient's blood pressure does not become sufficiently increased regardless of the increase in heart rate. Actual or relative central vascular volume depletion may occur because of gastrointestinal bleeding, dehydration, excessive diuresis, or the use of vasodilating drugs.

Following change in posture to the upright position, baroreceptors provoke an increase in medullary sympathetic outflow. This leads to vasoconstriction of the systemic resistance vessels and the splanchnic capacitance vessels. Compensation for continued orthostatic stress depends principally on the arterial baroreceptors. Disorders of abnormal autonomic vasomotor control leading to orthostatic hypotension may be primary or more commonly secondary. Primary pure autonomic failure and multiple system atrophy are both characterised by autonomic dysfunction, where the patient is unable to produce the appropriate vasomotor response following baroreceptor stimulation. ${ }^{16}$ Secondary autonomic disturbances leading to 
disturbance of blood pressure control include diabetic and alcoholic neuropathies, Addison's disease, paraneoplastic syndromes, and prolonged periods of physical inactivity. Those affected by dysautonomic syncope may suffer the classical fall of blood pressure of $20 \mathrm{~mm}$ $\mathrm{Hg}$ within three minutes of standing or they may have a progressive decline in blood pressure over a protracted period of time. ${ }^{16}$ Older patients are at particular risk of orthostatic hypotension and syncope because of altered baroreceptor responsiveness, polypharmacy, and the increased risk of volume depletion.

\section{Cardiac syncope}

Cardiac syncope results from inadequate effective cardiac output and may reflect serious underlying structural heart disease. A cardiac cause for syncope is an independent predictor of sudden death and mortality. ${ }^{2}$ The causes may be electrical (arrhythmic) or mechanical (obstructive). Patients with advanced heart failure and syncope have a one year mortality of $45 \%$, compared with $12 \%$ in patients without syncope. ${ }^{17}$

\section{ARRHYTHMIC SYNCOPE}

Rhythm disturbances are among the most frequent and potentially hazardous causes of syncope and dizziness. Syncope from arrhythmia most commonly results from ventricular tachycardia, which accounts for $11 \%$ of all cases of syncope. ${ }^{2}$ Those patients with depressed left ventricular function or myocardial ischaemia with or without infarction are at particular risk of ventricular tachycardia. The association of ventricular tachycardia with a low left ventricular ejection fraction cannot be overemphasised and must be considered in any patient with poor left ventricular function, whatever the aetiology. The haemodynamic consequences of ventricular tachycardia depend on the rate of the tachycardia and on cardiovascular autonomic tone. ${ }^{18}$ Syncope or presyncope caused by supraventricular tachycardia is believed to be associated with altered vasomotor tone independent of the tachycardia rate. ${ }^{19}$ Atrial fibrillation associated with the WolffParkinson-White (WPW) syndrome can lead to extremely rapid ventricular rates, causing syncope. Polymorphic ventricular tachycardia related to a long QT interval is a well recognised cause of syncope and sudden death.

In the older patient, bradyarrhythmias caused by sinus node dysfunction or conduction system disease are important causes of syncope. Sick sinus syndrome denotes a complicated rhythm disturbance involving impaired sinoatrial impulse formation or conduction. In patients with sick sinus syndrome, around $50 \%$ or more have the bradycardiatachycardia syndrome, usually manifesting as sinus bradycardia alternating with paroxysmal atrial tachycardia, atrial fibrillation, or atrial flutter. Patients with a sick sinus node or bradycardia-tachycardia syndrome may experience syncope from sinus arrest or following spontaneous conversion from a supraventricular tachycardia to sinus rhythm with a long
Box 2: Possible causes of syncope in aortic stenosis

- Inadequate increase in cardiac output and hence blood pressure maintenance because of fixed mechanical obstruction

- Arrhythmias

- Raised left ventricular systolic pressure giving rise to mechanoreceptor stimulation and hence neurally mediated syncope

- Concurrent degenerative disease of the atrioventricular node and His bundle leading to bradyarrhythmias

- Atrial tachyarrhythmias may substantially reduce ejection fraction in those with severe diastolic dysfunction

sinus pause. $^{20}$ Cardiac conduction system disease may represent acute or long standing cardiac ischaemia, cardiomyopathy, hypertension, valvar heart disease, or simply a chronic degenerative process. Persistent or episodic high grade atrioventicular block may result in sufficient loss of cardiac output to cause syncope. Severe bradycardia can leave the patient susceptible to ventricular tachyarrhythmias in association with a "pause dependent” long QT interval. ${ }^{21}$

MECHANICAL OBSTRUCTION AND OTHER CAUSES Obstruction to blood flow from the left ventricular outflow tract classically brings about syncope on exertion. This heralds a poor prognosis in severe aortic stenosis and warrants urgent valve replacement. Aortic stenosis may lead to syncope by various mechanisms (box 2). Young age and ventricular arrhythmias are highly predictive of syncope in patients with hypertrophic cardiomyopathy. ${ }^{22}$ Extreme outflow tract obstruction secondary to catecholamine stimulation and an association with the WPW syndrome are additional mechanisms for syncope in hypertrophic cardiomyopathy.

Recurrent pulmonary emboli can give rise to syncope in the absence of other symptoms. Syncope occurs in over $10 \%$ of patients with pulmonary embolism and is more likely to occur with large emboli. Massive pulmonary embolism can obstruct the flow of blood in the pulmonary artery leading to reduced cardiac output. ${ }^{23}$ Smaller pulmonary emboli can give rise to vagally mediated bradyarrhythmias; these occur because of activation of pulmonary or ventricular stretch receptors, in an analogous fashion to neurocardiogenic syncope. ${ }^{24}$

Myocardial infarction is most likely to present atypically as syncope in the elderly; probably because of transient bradyarrhythmias resulting from a reflex bradycardia (the Bezold-Jarisch reflex); otherwise it is a rare direct cause of syncope. ${ }^{6}$ Other mechanical cardiovascular causes of syncope are relatively rare, such as left atrial myxoma, severe mitral stenosis, or prosthetic valve dysfunction. 


\section{Summary points}

- The commonest form of syncope, the "vasovagal" faint, is associated with a good prognosis.

- Elderly people are susceptible to acute changes in cerebral blood flow, predisposing them to syncope.

- Syncope in the presence of structural heart disease and a low left ventricular ejection fraction is associated with a high mortality rate.

\section{Neurological and psychiatric diseases}

In the absence of accompanying focal neurological symptoms and signs, syncope from cerebrovascular disease is rare. Transient ischaemic attacks caused by vertebrobasilar insufficiency may cause syncope. Those affected tend to be elderly men with ischaemic heart disease. Concurrent neurological symptoms include mainly vertigo, ataxia, or sensory disturbances. ${ }^{25}$ Transcranial Doppler ultrasonography has been used during head up tilt testing to demonstrate cerebral vasoconstriction associated with syncope that precedes, or even occurs in the absence of, systemic hypotension. ${ }^{926}$ This phenomenon has been termed cerebral syncope.

Distinguishing seizures from syncope can be difficult, especially if a patient experiences "convulsive syncope." Convulsive movements, similar to tonic-clonic seizure activity, can occasionally result from cerebral hypoxia secondary to cerebral hypoperfusion. While neurally mediated syncope may mimic seizure-like activity, it should also be acknowledged that seizure foci in certain cerebral sites (particularly the temporal lobe) may be the source of apparent neurally mediated syncopal events. Localised seizure activity may initiate the reflex arc previously described, leading to hypotension and bradycardia.

The prevalence of psychiatric illness in patients with syncope of unknown origin is around $24 \%{ }^{27}$ Hyperventilation, particularly in panic disorder, leads to hypocapnia, causing a transient increase in cerebrovascular resistance coupled with simultaneous peripheral vasodilatation. Vasovagal syncope can be caused by acute stress or fear and is therefore implicated in anxiety, panic, and major depressive disorders. Certain individuals continue to have recurrent unexplained syncope despite thorough investigation. Some patients may actually experience syncope during tilt testing, with no measurable change in blood pressure, heart rate, EEG pattern, or transcranial blood flow. ${ }^{9}$ This finding has been termed "psychogenic syncope" and is believed to be a somatoform disorder.

1 Kapoor WN. Evaluation and management of the patient with syncope. $\mathcal{F} A M A$ 1992;268:2553-60

2 Kapoor WN. Evaluation and outcome of patients with syncope. Medicine 1990;69:160-75.

3 Manolis AS, Linzer M, Salem D, et al. Syncope: current diagnostic evaluation and management. Ann Intern Med 1990;112:850-63.

4 Lipsitz LA, Wei JY, Rowe JW. Syncope in the elderly, institutionalised population: prevalence, incidence, and associated risk. $Q \mathcal{F} M$ Med 1985;55:45-55.

5 Gowers WR. A lecture on vagal and vasovagal attacks. Lancet 1907;173:1551-3.

6 Wayne HH. Syncope: physiological considerations and an analysis of the clinical characteristics in 510 patients. $A m \mathcal{F}$ Med 1961;30:418-38.

7 Benditt DG. Neurally mediated syncopal syndromes: pathophysiological concepts and clinical evaluation. PACE 1997;20:573.

8 Shalev Y, Gal R, Tchou PJ, et al. Echocardiographic demonstration of decreased left ventricular dimensions and vigorous myocardial contraction during syncope induced by head-up tilt. $\mathcal{F}$ Am Coll Cardiol 1991;18:746-51.

9 Grubb BP, Kosinski D. Tilt table testing: concepts and limitations. PACE 1997;20:781-7.

10 Abboud FM. Neurocardiogenic syncope. $N$ Engl f Med 1993;328:1117-1120

11 Sutton R, Peterson M, Raviele A, et al. Proposed classification for tilt-induced vasovagal syncope. Eur $\mathcal{f}$ Cardiac Pacing Electrophysiol 1992;2:180-3.

12 Abi-Samra F, Maloney JD, Fouad-Tarazi FM, et al. The usefulness of head up tilt testing and hemodynamic investigations in the work up of syncope of unknown origin. PACE 1988;11:1202-14.

13 Thomas JE. Hyperactive carotid sinus reflex in carotid sinus syncope. Mayo Clin Proc 1969;44:127-39.

14 Wagshal AB, Huang SKS. Carotid sinus hypersensitivity. In: Grubb BP, Olshansky B, eds. Syncope: mechanisms and management. Armonk, NY: Futura, 1998:281-95.

15 Gaggioli G, Brignole M, Menozzi C, et al. Reappraisal of the vasodepressor reflex in carotid sinus syndrome. Am 7 Cardiol 1995;75:518-21.

16 Mathias CJ. The classification and nomenclature of autonomic disorders: ending chaos, resolving conflict and hopefully achieving clarity. Clin Auton Res 1995;5:307-10.

17 Middlekauff HR, Stevenson WG, Saxon LA. Prognosis after syncope: impact of left ventricular function. Am Heart $\mathcal{f}$ 1993; 125:121-7.

18 Huikuri HV, Zaman L, Castellanos A. Changes in spontaneous sinus node rate as an estimate of cardiac autospontaneous sinus node rate as an estimate of cardiac auto-
nomic tone during stable and unstable ventricular tachycardia. F Am Coll Cardiol 1989;13:646-52.

19 Leitch JW, Klein GJ, Yee R, et al. Syncope associated with supraventricular tachycardia: an expression of tachycardia rate or vasomotor response? Circulation 1992;85:1064-71.

20 Moss AJ, Davis RJ. Brady-tachy syndrome. Prog Cardiovasc Dis 1974;16:439-545.

21 Jackman WM, Friday KJ, Anderson JL, et al. Idiopathic long QT syndrome: a critical review, new clinical observations and a unifying hypothesis. Prog Cardiovasc Dis 1988;31:11572 .

22 Nienaber CA, Hiller S, Spielman RP, et al. Syncope in hypertrophic cardiomyopathy: multivariate analysis of prognostic determinants. $\mathcal{f}$ Am Coll Cardiol 1990;15:948-55.

23 Thames MD, Alpert JS, Dalen JE. Syncope in patients with pulmonary embolism. fAMA 1977;238:2509-11.

24 Simpson RJ, Podolak R, Mangano CA, et al. Vagal syncope during recurrent pulmonary embolism. $\not A M A$ 1983;249: during

25 Davidson E, Rotenbeg Z, Fuchs J, et al. Transient ischemic attack-related syncope. Clin Cardiol 1991;14:141-4.

26 Grubb BP, Samoil D, Kosinski D, et al. Cerebral syncope: loss of consciousness associated with cerebral vasoconstriction in the absence of systemic hypotension. Pacing Clin Electrophysiol 1998;21:652-8.

27 Jeong H, Kapoor W. Psychiatric illness and syncope. Cardiol Clin 1997;15:269-75. 